\title{
Computation of Equilibrium Paths in Nonlinear Finite Element Models
}

\author{
Zdeněk Kala ${ }^{1, a}$ \\ ${ }^{1}$ Brno University of Technology, Department of Structural Mechanics, Veveři 95, 60200 Brno, Czech Republic
}

\begin{abstract}
In the present paper, equilibrium paths are simulated applying the nonlinear finite element model. On the equilibrium paths, there are identified critical points which contribute to understanding and quantification of stability processes in a nonlinear system. By means of nonlinear solution, bifurcation points from which two equilibrium path branches emanate and so there is no unique tangent were identified and drawn. Mutual position of limit and bifurcation points was identified. The paper describes multidisciplinary problems of the analysis of limit states of nonlinear systems. The methods of stochastic and sensibility analyses which are frequently applied to assessment of the safety and reliability of supporting structural systems are discussed.
\end{abstract}

\section{Introduction}

The stability of slender bars is a phenomenon associated with buckling [1]. Even though the failure mechanism of a steel bar may appear to be a fundamental problem, its basis has often eluded researchers and practitioners. No matter how slender a steel bar, it does not reach its ultimate static resistance (load-carrying capacity) under elastic buckling, but rather after sufficient yielding of the critical cross-section under combined compression and bending [2].

In reality, steel bars are not perfectly straight, but are invariably subjected to some degree of initial crookedness. The shape of the steel bar is typically defined by the path denoted as "Imperfect bar" plotted in Fig. 1. Results of theoretical and experimental research present an important source of data for sensitivity analyses [3-6], assessment of structural reliability [7-10], and for multiple-criteria decision analyses, see, e.g., [1116]. Results of experimental research of the loaddeflection path have been published, e.g., in [17-19].

Specific scenarios of post-buckling behaviour are frequently attributed to certain structural types, like columns, shells or plates [1]. Structural post-buckling response has strong implications on the structural load carrying capacity, sensitivity to imperfection, structural safety, and is of great significance to current design procedures [1].

The nonlinear finite element solution performed using current software is, as a result of the instabilities of high von Mises truss, numerically demanding, and discrepancies could exist between the obtained result and experimental results [19]. In general, the phenomenon of loss of stability is present in the deforming structure regardless of whether the loading process is controlled by force $[5,7]$ or by displacement [20]. The geometrically nonlinear solution performed on the basis of increasing the deflection reliably investigates the full path of beam imperfection, see Fig. 1. Upon reaching the peak, the force decreases and the deformation increases. Reaching the peak of the load-deflection path can result in numerical problems for common software based on the geometrically nonlinear finite element solution, especially in cases, when the loading process is controlled by load increments.

The geometrically nonlinear finite element method, which has proven to be suitable for solving stability types of problems of the von Mises trusses [21], was applied in the presented article. The system load-deflection paths were evaluated using this method. The resulting outputs of the geometrically nonlinear solution are the static equilibrium states.

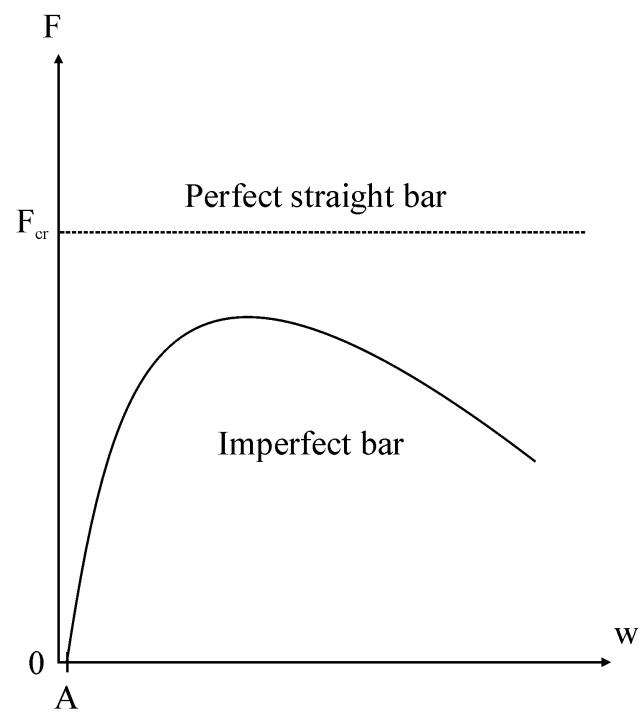

Figure 1. Load-deflection path of pin-ended model.

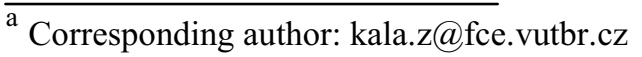




\section{Bifurcation and limit points}

Light weight steel structures often work in a nonlinear range; this is due to always increasing requirements for low price and high strength. The stability analysis of slender steel members is the key aspect of safety and reliability of constructional solution. The structure stability is often determined by singular points in its equilibrium path. These points can represent severe problems for common geometrically nonlinear finite elements methods based on increase of load actions which are routinely applied to engineering designs [2224].

Each point of an equilibrium path represents an equilibrium state. Along a static equilibrium path of a conservative system, transition from stability to instability can occur at critical points only. Critical points are divided into limit and bifurcation points, see Fig. 2 and Fig. 3.

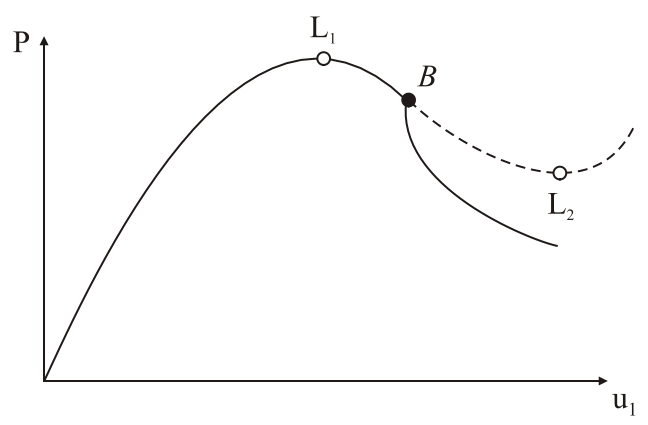

Figure 2. $P$ vs $u_{l}$ plane: Limit point before bifurcation.

The parameter $P$ which controls the structural behaviour in dependence on displacement $u_{1}$ is introduced to vertical axes. The identification of critical points is well known [24]. When applying the finite elements method, critical points can be identified using tangent stiffness matrix $K$. The matrix $K$ is symmetric and real. The critical point takes place at the moment when matrix $K$ is singular. The value of determinant of matrix $K$ is the fundamental criterion for the identification of critical points. The isolated critical point has only one zero eigenvalue of matrix $K$. The multiple critical point has two or more zero eigenvalues of matrix $K$. The structure with multiple critical point is more sensitive to imperfections in the vicinity of that critical state [25]. The matrix of toughness $K$ of the regular point of equilibrium path is nonsingular.

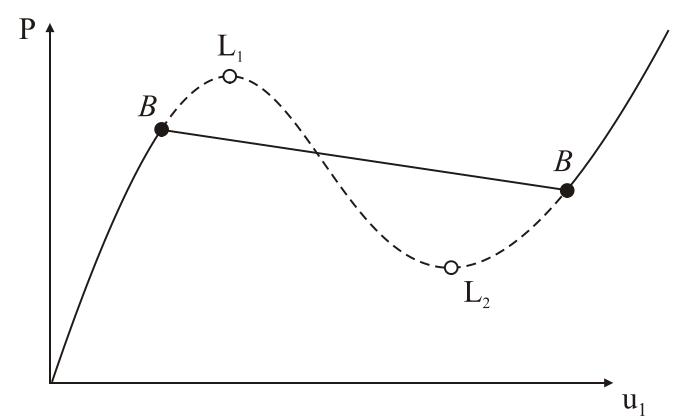

Figure 3. $P$ vs $u_{l}$ plane: Bifurcation point before limit point.
Bifurcation point, also called branch point or branching point, is point from which two equilibrium path branches emanate and so there is no unique tangent. Bifurcation of equilibrium is characterized by the sudden transition from one deformation regime to another regime.

General understanding of the basic characteristics of the elastic buckling and postbuckling behaviour of members that become unstable as a result of bifurcation can be obtained by considering the simple model in Fig. 4. The object of the present paper is computing of equilibrium paths of pin-ended slender elastic von-Misses truss. The imperfect truss was considered as symmetrical, so only one half of the structure is plotted, see Fig. 4. The determination of failure load of the slender strut requires to take the influence of initial curvature of the bar axis into consideration and to consider the entire nonlinear load deflection curve of the structure, see Fig. 4.

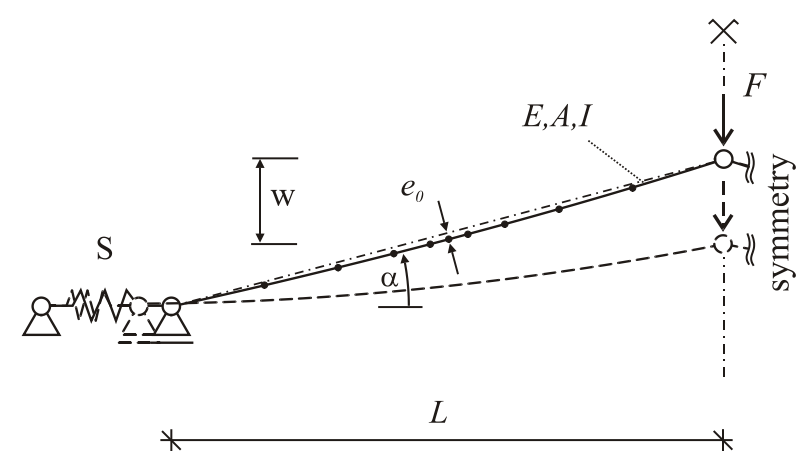

Figure 4. Bifurcation-buckling model of imperfect system.

\section{Post buckling analysis}

The planar truss with member HEA 200 and initial angel $\alpha=15^{\circ}$ was analyzed, see Fig. 4. The HEA 200 is the cross-section with second moment of area $I_{z}=13.36 \mathrm{E} 6$ $\mathrm{mm}^{4}$ and section area $A=5380 \mathrm{~mm}^{2}$. Young's modulus is $E=210 \mathrm{GPa}$. The bar has initial imperfection of bar axis. The one half sine function with aplitude $e_{0}$ was used for this imperfection. The computing of equilibrium paths of these geometrically nonlinear systems was solved using the software created by the author of the present paper, see Fig. 5.

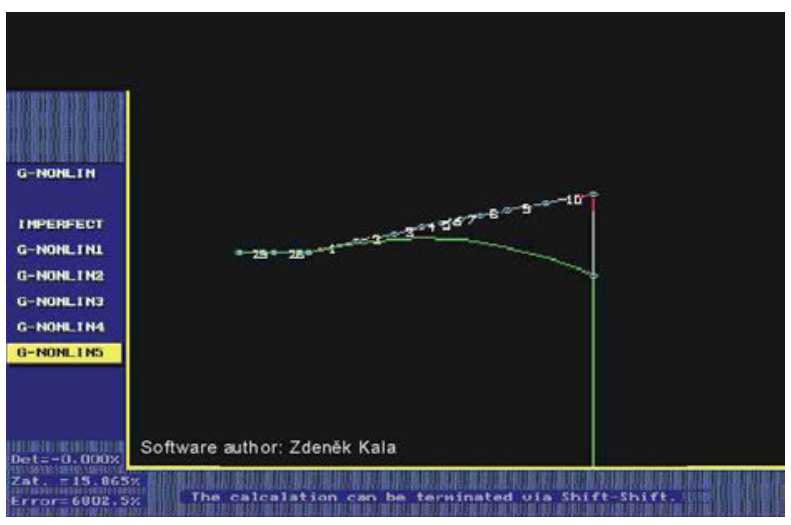

Figure 5. Computer program for bifurcation-buckling analysis. 
The full-line black curve in Fig. 6 corresponds to the truss with $e_{0}=0$ (without initial imperfections). During increasing the load we reach the point "A" as the top of load-deflection curve. If we increase the load, the jump must occur and the displacement will be fixed in the point "D". Then, we can continue increasing the load. The jump is the so called snap-trough effect [26]. Buckling in the bar with $e_{0}=0$ will occur by bending about minor axis in the moment when the axial force in bar is equal to the Euler critical force $N_{c r}=1.54 \mathrm{MN}$. The bifurcation point at which buckling will occur, is marked by the black point "B", see Fig. 6.

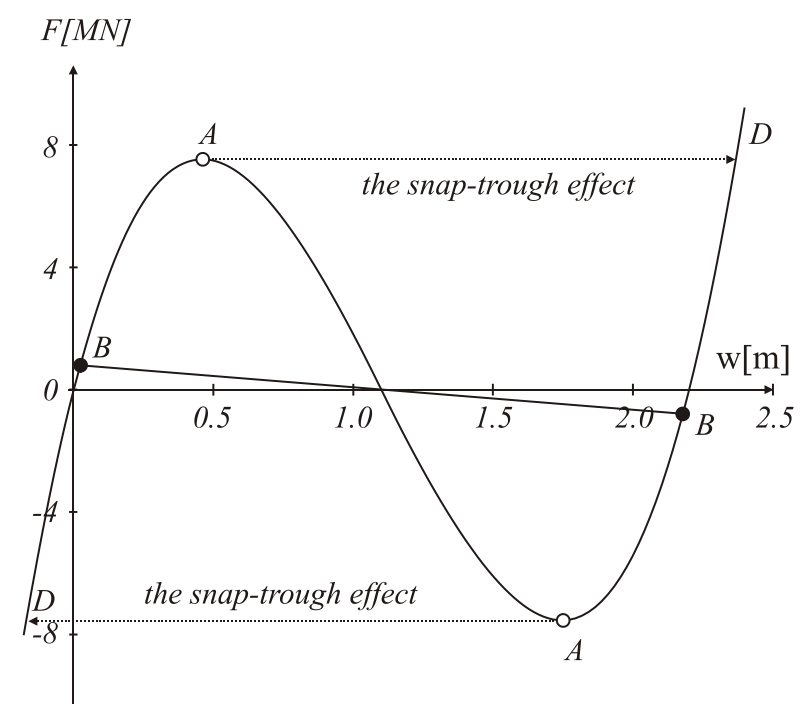

Figure 6. The snap-trough effect of von Misses truss.

After the bifurcation point, there follows the bifurcation of equilibrium, based on which buckled or unbuckled configuration of bar deformation is possible. Limit points of imperfect $\left(e_{0}>0\right)$ bars are illustrated in hollow manner on dashed lines, see Fig. 7.

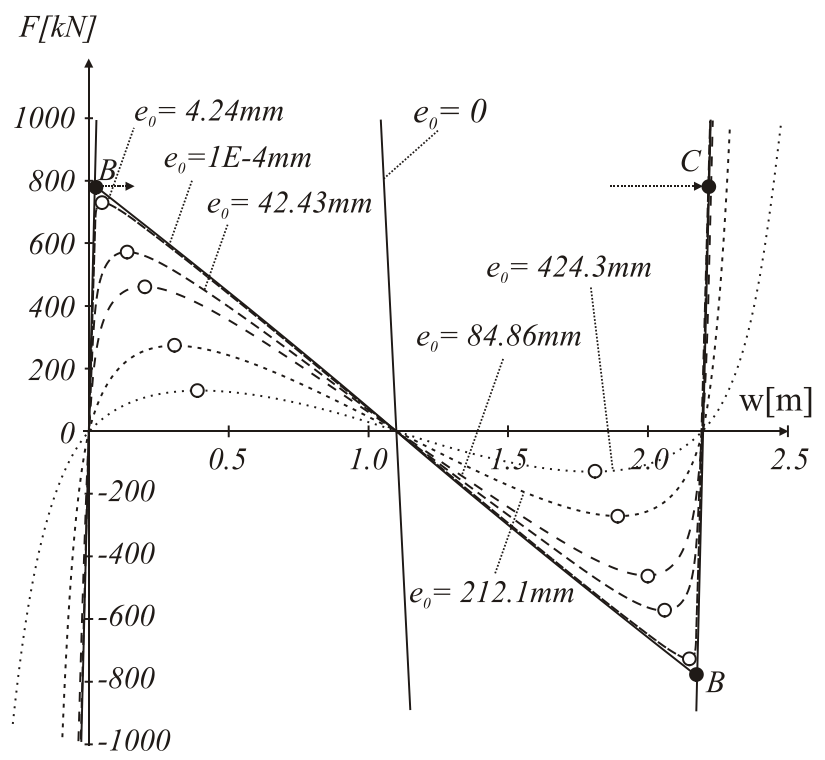

Figure 7. Load-deflection curves for $S=\infty$.

The bifurcation point and limit points lie on the straight line. After buckling, there will take place the snap from the point $\mathrm{B} \rightarrow \mathrm{B}$ due to the negligible increase of force $F$ or there will be decreasing $F$ due to increasing $w$, see Fig. 7. Snap-through instability mechanism of structure deformation is unstable under force $F$ control of loading but it is stable under displacement $w$ control of loading.

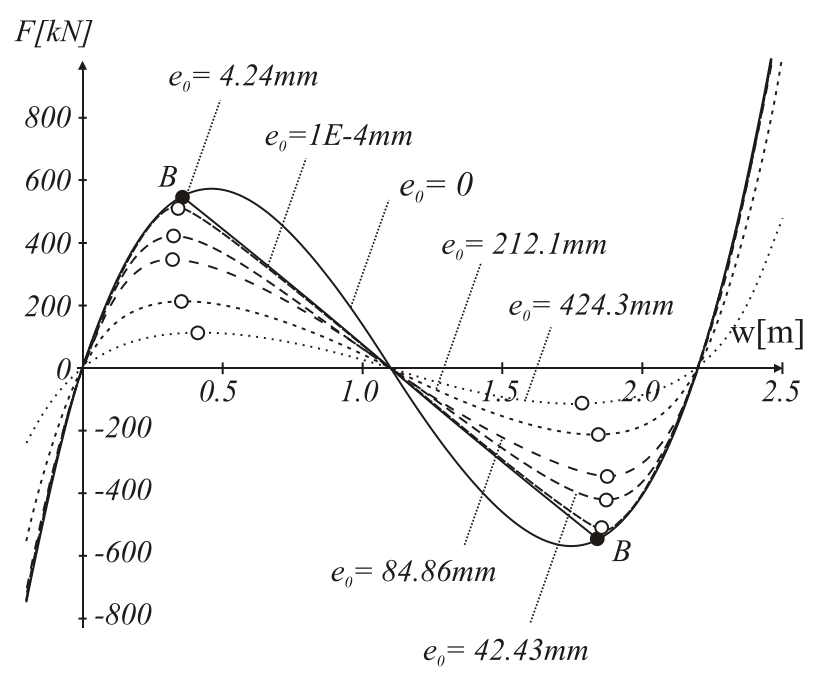

Figure 8. Load-deflection curves for $S=21.21 \mathrm{MNm}^{-1}$.

The full-line black curve in Fig. 8 corresponds to the truss with $e_{0}=0$ (without initial imperfections) for stiffness $S=21.21 \mathrm{MNm}^{-1}$. The bifurcation point and limit points are not presented on the straight line. The connection of configuration both of bifurcation point and limit points with initial imperfection and stiffness $S$ can be analysed. When comparing Fig. 7 with Fig. 8 , it is evident that the bifurcation point and limit points change their configuration from the position on the straight line to the situation on the curve. Finding dependences between $e_{0}, S$ and limit points forms the basis for the analysis of equilibrium paths without application of nonlinear finite elements models.

\section{System stability and randomness}

A stable structure is the one which remains stable for any conceivable (imaginable) system of loads. The stability is mostly examined on a structure without imperfections. The result of common stability analysis is the value of critical loading at which the structure buckles. If the critical load is remarkably higher than the load action of structure, then the structure service is reliable and safe. Stability analysis should be carried out for all load action states, which can be counted up to thousands. Engineering computation and evaluation of results can be complicated, in particular if there occur multiple critical points. A large number of loading states in real structures represents a complication which is overcome by the engineers by means of approximate methods.

\subsection{Buckling length}

The static resistance of bars under compression stress can be computed by using the so-called buckling length. 
Buckling length is represented by the structure parameters which take into consideration the influence of initial imperfections on the resistance decrease. Although there exist sophisticated approaches how to calculate the buckling length, it is always the engineer who estimates the structure as for ultimate limit states, who is responsible for their resulting choice. The occurrence of multiple critical points can cause the incorrect and dangerous assessment of buckling lengths. An alternate parameter to the buckling length is the slenderness of the bar, for the application of which the same cautiousness is valid as that for buckling lengths.

\subsection{Failures and stability phenomenon}

Many failures of building structures are caused by the stability loss. Many times repeated loading, which causes repeated buckling of slender elements and fatigue crack, is dangerous above all [27]. This phenomenon is met in bridge structures. Safety and reliability of slender bridge structures gets degraded in time, and enforces regular inspection checking up. The lifetime of bridge structures must be always specified, based on results of each inspection checking up which helps detect fatigue cracks and other failures occurring in bridges.

\subsection{Imperfections and initial imperfections}

In literature, the notions "imperfection" [28] and "initial imperfections" [3-5] are frequently met. In general, material and geometrical imperfections are distinguished [29]. Whereas an imperfection can be both small and large, an initial imperfection is assumed, as a rule, to be very small, in particular when geometrical imperfections are concerned. The size of initial imperfections always influences the safety and reliability of structures. Ultimate limit states are sensitive to the variability of initial imperfections less than serviceability limit states.

\subsection{Stochastic computational models}

The general development of theory and methods of design of structures is remarkable due to its multidisciplinary approach the tendency of which is the step by step transition from deterministic approach through semiprobabilistic conception to the direct probabilistic computation method. The development of computer technology enables to elaborate numerically demanding computer simulations of nonlinear systems based on computer modelling, non-linear systems, stochastic systems, neural networks, fuzzy systems, evolutionary computation, systems theory, soft computing, lightwave engineering and mathematical theory of stability. Stochastic calculation models consist of the deterministic nonlinear computation model (usually based on the nonlinear finite elements method), and on the numerical simulation method of Monte Carlo type. It is not possible to find the real value of structure failure probability; therefore it is being worked with its theoretical value. The calculation of probability of structure failure is always possible with a certain accuracy only. Therefore it is frequently spoken on the assessment of failure probability which testifies to the structure reliability, and provides information for decision makers. The lower fuzzy uncertainty of input data and of parameters of computation models, the more credible can be the assessment of structure reliability.

\subsection{Sensitivity analysis}

Sensitivity analysis is the crucial part of sophisticated computation models the influences of input quantities on the model output of which cannot be understood by simple intuition. Research [3-7] showed that the evaluation of the ultimate limit state by the sensibility analysis based on correlation between load carrying capacity and geometrical imperfection would lead to incorrect results. It is due to the fact that the imperfection decreasing or increasing from zero decreases the load carrying capacity.

The dependence and the correlations cannot be interchanged, nevertheless, a correlation can be chosen as the sensitivity indicator of the first choice in numerically demanding nonlinear computation models which other sophisticated approaches cannot be applied to.

\section{Conclusion}

In the paper, there were identified load-deflection curves of a geometrically nonlinear model with imperfections, and problems of multiple critical points were discussed. The probability of occurrence of multiple critical points can indicate high sensitivity of response of the system to initial imperfections [25]. The basic methods of stability analysis of nonlinear systems used in engineering practice were described in the present paper. The failures of slender structural system caused by stability loss frequently cause the decrease of safety and reliability. Modern methods of stability analysis are important instruments to ensure function reliability and general effectiveness of modern structural systems.

\section{Acknowledgment}

This result was achieved with the financial support of the projects No. LO1408 “AdMAs UP" and GA15-07730S and FAST-S-16-3779.

\section{References}

1. T.V. Galambos, Stability Design Criteria for Metal Structures, 911 (1998)

2. L.H. Teh, B.P. Gilbert, J. Constr. Steel. Res. 117, (2016)

3. Z. Kala, J. Kala, WSEAS Transactions on Applied and Theoretical Mechanics 4, 3 (2009)

4. Z. Kala, Arch. Civ. Mech. Eng. 15, 4 (2015)

5. Z. Kala, J. Civ. Eng. Manag. 22, 3 (2016)

6. R. Sousa, J. Guedes, H. Sousa, Arch. Civ. Mech. Eng. 15, 2 (2015) 
7. Z. Kala, J. Kala, A. Omishore, International Journal of Mechanics 10, (2016)

8. Z. Kala, A. Omishore, International Journal of Mathematics and Computers in Simulation 10, (2016)

9. A. Omishore, Proc. of the 4th WSEAS Int. Conf. on EMESEG'11, pp.284-288 (2011)

10. M. Kvíčala, K. Frydrýšek, M., Štamborská, International Journal of Thermophysics 36, 2-3 (2015)

11. J. Antucheviciene, Z. Kala, M. Marzouk, E.R. Vaidogas, Math. Probl. Eng. 2015, 362579 (2015)

12. J. Antucheviciene, Z. Kala, M. Marzouk, E.R. Vaidogas, Math. Probl. Eng. 2015, 160569 (2015)

13. F. Baño, Á. Mena, F. Viscaino, J. Rubio, G. Rodríguez-Bárcenas, International Journal of Economics and Statistics 4, (2016)

14. E. Castillo, J.A. Lozano-Galant, M. Nogal, J. Turmo, J. Civ. Eng. Manag. 21, 6 (2015)

15. D.B. Hammad, N. Shafiq and M. F. Nuruddin, MATEC Web of Conferences 15, 01018 (2014)

16. M. Medineckiene, E.K. Zavadskas, F. Björk, Z. Turskis, Arch. Civ. Mech. Eng. 15, 1 (2015)

17. M. Štrba, M. Karmazínová, International Journal of Mechanics 9, (2015)

18. M. Karmazínová, J. Melcher, WSEAS Transactions on Applied and Theoretical Mechanics 9, (2014)

19. O. Sucharda, J. Vasek, J. Kubosek, International Journal of Mechanics 9, (2015)

20. Z. Kala, M. Kalina, International Journal of Mechanics 10, (2016)

21. Z. Kala, J. Civ. Eng. Manag. 18, 1 (2012)

22. V. Zakovorotny, WSEAS Transactions on Applied and Theoretical Mechanics 10, (2015)

23. J. Shi, Computers \& Structures 58, 1 (1996)

24. Z.P. Bažant, L. Cedolin, Stability of structures: Elastic, inelastic, fracture and damage theories, (1991)

25. Z.P. Bazant, Y.Y. Xiang, Journal of Structural Engineering ASCE 123, 4 (1997)

26. J. Ravinger, Stability \& Vibration, 135 (2012)

27. Z. Kala, J. Kala, M. Škaloud, B. Teplý, J. Civ. Eng. Manag. 11, 2 (2005)

28. A. Steinboeck, X. Jia, G. Hoefinger, H.A. Mang, Comput. Meth. Appl. Mech. Eng. 197, 45-48 (2008)

29. Z. Kala, Eng. Struct. 31, 6 (2009) 